\title{
Paper in Architecture: The role of additive manufacturing
}

\author{
T. Campos \\ School of Architecture, University of Minho, Guimarães, Portugal \\ P. J. Cruz \& B. Figueiredo \\ Lab2PT, School of Architecture, University of Minho, Guimarães, Portugal
}

\begin{abstract}
The introduction of Additive Manufacturing (AM) brought transformative approaches for the building industry by the development of new systems and the exploration of new components with complex geometries. This paper intends to demonstrate the different AM Techniques in Architecture by mixing different materials, such as cellulose and starch. The research focus on the ecological potentials and limitations of a completely biodegradable material, cellulose - polysaccharide present on plant walls - for architecture using 3D printing techniques. The sensitive nature of this project is to take advantage of the potential of cellulose, an abundant, recyclable and biodegradable material, while transforming into a pulp for application in 3D printing for generation of new models taking advantage of the potentialities of the techniques in AM.
\end{abstract}

\section{INTRODUCTION}

In the contemporary context, the work of the Japanese architect Shigeru Ban is paradigmatic in the integration of building components made of paper, where this material is present on the architectural expression of the building, revealing the potential of paper as building material (Latka 2017).

Nowadays the technology and the exploration of innovative materials, expand the way in which pulp can be integrated into the contemporary architecture. Cellulose is one of the most valuable materials and the main component of the plants used for the production of paper, the extraction of cellulose in its fibrous state is the basic process for the production of a pulp.

This paper describes a research which aims to analyse the use of mixtures based on cellulose in Additive Manufacturing (AM) processes, that is to infer their potential and constrains. Essentially, the research focuses on the mix of two raw materials, cellulose and clay, according to the organization of this article. The work initiated by the exploration of mixtures based on cellulose and starch (section 3), followed by studies of mixtures of cellulose and clay (section 4). This study allowed us to obtain conclusions by comparing the behaviour of cellulose pulp.

\section{ADDITIVE MANUFACTURING CONTEXT}

The AM technique used on this research was Liquid Deposition Modeling (LDM). This process consists in the continuous deposition, or extrusion, of layers of viscous materials (Rosenthal et al., 2017). The 3D printer used on the production of specimens and prototypes moves an extrusion nozzle and cartridge. The extrusion flow is controlled by a spindle, and the air pressure applied to the cartridge filled with the material. The CAD-CAM tool used to generate the GCode was developed by our team in order to fully control the manufacturing process (Barbosa and Figueiredo, 2016). The application was implemented trough Grashopper ${ }^{\circledR}$ - an application programming interfaces of the 3D modelling CAD software Rhinoceros ${ }^{\circledR}$ that uses Visual Programming Language that allows to customise the extrusion path, speed, layer thickness, layer height and the creation of structures to support the object when printed (Figure 1).

Specimens, prototypes and tests produced in the research presented in this paper were made on the Advanced Ceramics R\&D Lab (ACLab), located at the Institute Design of Guimarães, University of Minho, having taken advantage of the existing resources.

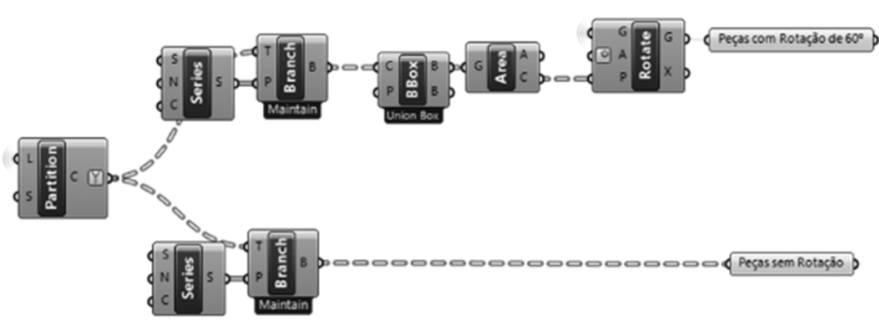

Figure 1. Grasshopper ${ }^{\circledR}$ interface illustrating the generation of the G-Code data. 


\section{CELLULOSE, A RAW MATERIAL FOR AM}

Cellulose is an abundant polymer on the walls of plants. To study these fibers it is necessary to take into account the basic principles of the anatomy of the wood, it's identification according to the type of species. Cellulose is a fiber with application in the most diverse areas of production, such as food, but also on the composition building materials. Cellulose is the main structural fiber of the plant Kingdom, being composed by a long chain polymer classified as polysaccharide or carbohydrate. In the words of Klemm et al. (2005), cellulose is the most common organic polymer and is considered as an almost inexhaustible source of raw material for the increasing demand for environmentally friendly and biocompatible products (Latka 2017).

Two types of cellulose pulps used in the industry of paper and transformation were consider in this study: cellulose pulp in plates (Figure 2) and cellulose pulp bleached powder (Figure 3).

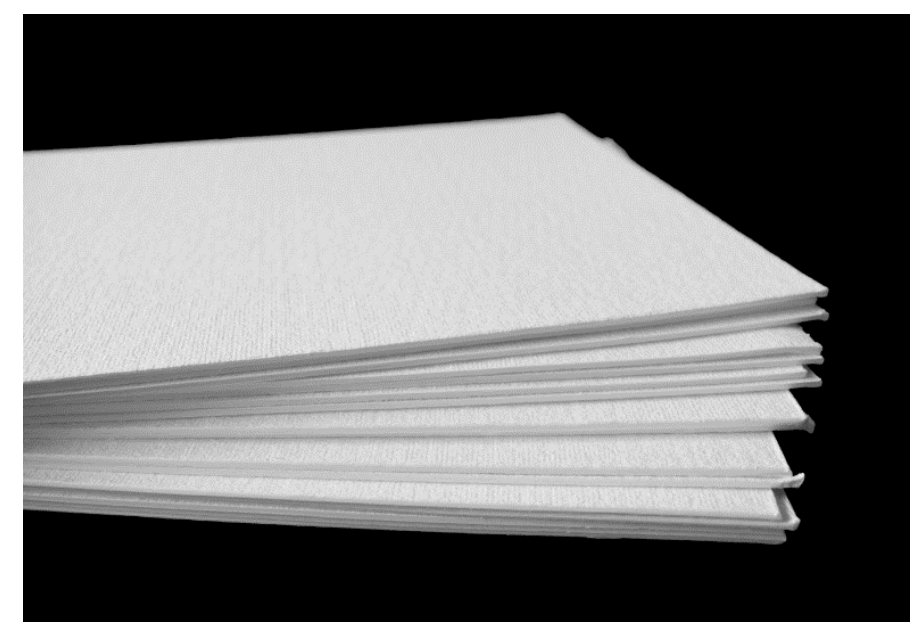

Figure 2. Cellulose paste bleached in plates.

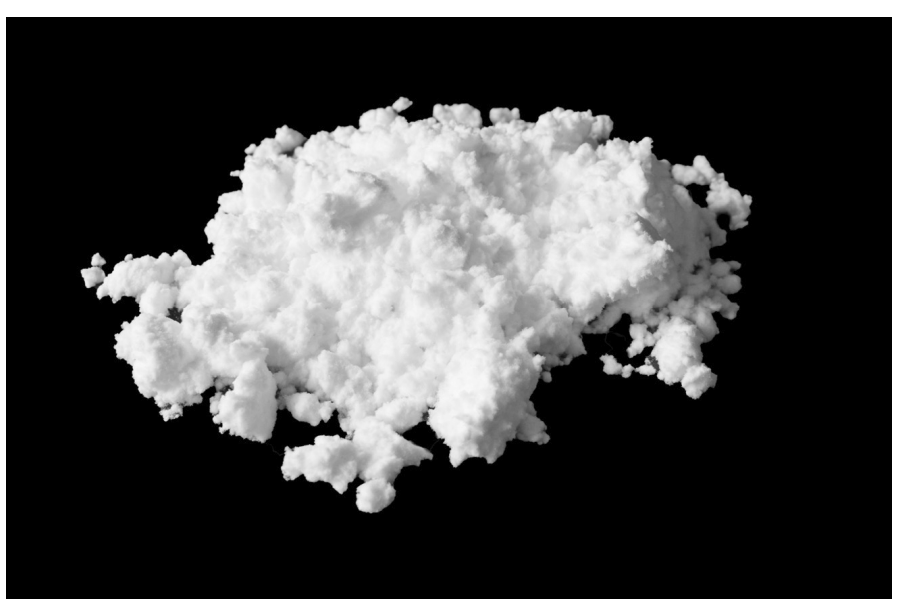

Figure 3. Cellulose paste bleached powder.

\section{STARCH AND CELLULOSE}

\subsection{Introduction}

As already mentioned, the main goal of this research was to infer material compositions of cellulose pulp with adequate behaviour for LDM process. The methodology adopted took three phases of work, the first phase was focused on the research of possible materials to combine with the cellulose that could reach the plasticity needed for the extrusion. Materials such as acetone, agar-agar, white glue and starch were used, but only starch presented workability for application in LDM. The main goal of this phase was to reach a pulp composition that presents a plasticity similar to clay mixtures with successful degree of viscosity for AM for extrusion. The second phase, after observing the behaviour of the materials used in the first phase, consisted in studying in detail the combination between cellulose and starch which generated a reason for the mixing. The starch needs to be mixed with water and then heated at low heat to form a gelatinous pulp. To create this mixture two cellulose pulps were analysed, one in plates, which needs to be previously mixed with water to obtain small grains of cellulose and a second one in powder. The third phase resulted in the application of pulp composed by cellulose and starch to 3D printing. Individual studies were carried out to each of the pulps with the aim of analysing parameters such as plasticity, validity, workability and strength for AM. The last stage consisted in the design and manufacture of a prototype wall composed by hexagonal blocks where different composite materials were tested.

Combining the materials and generating the pastes from the different materials, pulp of cellulose in plates, pulp of cellulose powder, starch and wood fibers, the main objective was the study of its properties, such as the plasticity of the pulp, the workability when applied in AM, the resistance in the production of prototypes and architectural components and their final appearance. The following sections of the paper describe in greater detail the different idealized mixtures and their behaviour when applied in AM.

\subsection{Starch}

Starch is a carbohydrate present in plants, when it comes into contact with water it dissolves and if subsequently boiled, it can transform into a gelatinous material, giving plasticity to the mixture when coupled to another material. It's the change from liquid to gelatinous state is its greatest advantage as a material for application in AM, because it confers a strong bond between the multiple cellulose particles. For the transformation of the material 50 grams of starch is required in 350 grams of hot or cold water. This preparation requires some care in its processing time so in order to preserve its characteristics, either in the 
weighing of the materials, so that the weight of attached starch is in conformity with the amount of water present.

\subsection{Mixture A}

The mixture A, composed by starch and cellulose pulp in plates was the first to be thought and the one that more fields of investigation potentiated for the accomplishment of the following ones. The cellulose pulp (Figure 2) is a raw material that needs to be processed before being mixed with other materials. For this transformation to occur, the pulp needs to be dissolved in water until the formation of small grains of cellulose. In order to generate the mixture A, it is necessary to follow only two steps:

1. The weighing of 350 grams of cellulose pulp;

2. Attach 150 grams of previously boiled starch, aggregation of both materials.

In order to start the study, a set of parameters was defined with the goal of studying and validating the mixture A. These parameters are divided into two phases, (1) the validation of the mixture for application in the AM and (2) its workability for the composition of architectural elements. These steps were applied throughout all of the defined mixtures. All the results obtained in the different tests and specimens produced were observed, being possible to infer some conclusions. In order to validate the use of the mixture in AM it was performed a simple test of extruding small circles. In the various small circles different parameters were tested, variation in the air pressure, differentiation of fluidity and diameters of the extrusion nozzles. In order to evaluate the workability of the mixture for application and production of architectural elements, different specimens with different wall curvatures were developed. The tests produced allowed to verify geometric constrains when the mixture was used in the LDM technique (Figure 4).

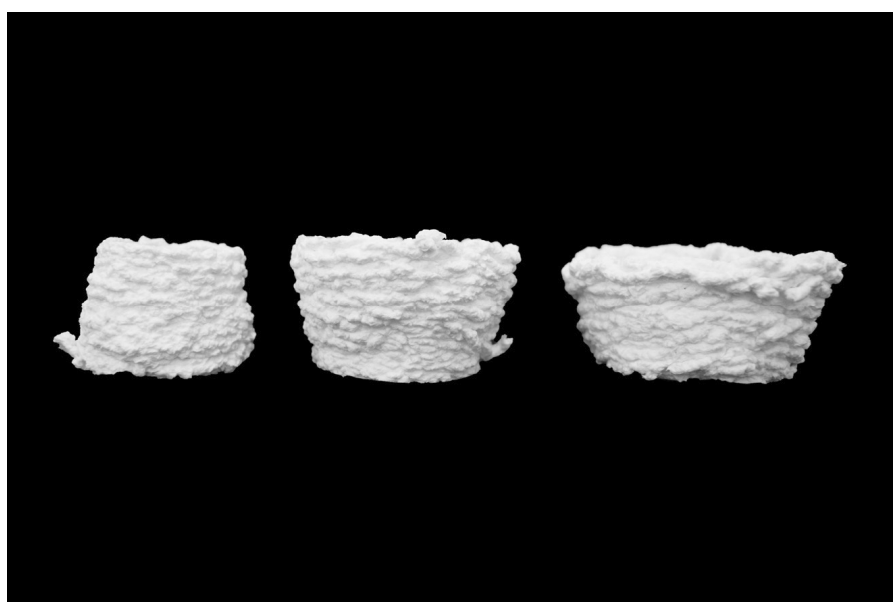

Figure 4.Models printed with mixture A, with different inclinations, to study the curvature limitation.

\subsection{Mixture $B$}

Composed of starch and cellulose pulp powder, the mixture $\mathrm{B}$ was the one that contributed the most for the development of this research. Similarly mixture A, to achieve mixture $\mathrm{B}$, it is necessary to follow two steps:

1. The weighing of 60 grams of cellulose pulp;

2. Package of 300 grams of previously boiled starch and aggregation of both materials.

When the mixture A was studied, the major problems encountered during its use for AM were related to the final shape of the mixture, when the cellulose grains are saturated with water, the fluidity of pulp is excessive, if the grains are dry, they generated problems in the printing process. All problems previously encountered with the use of the processed cellulose plates were solved by simply attaching powder instead of grains to the mixture.

As described in the mixture A, some study parameters were defined for the validation of the mixture for AM.

The first test consisted on the manufacture of three single pieces, one cylinder and two truncated cones. After verification of the workability of the mixture B, a second test was carried out, such as in mixture A cellulose in plates and starch printed different models with different wall inclinations. The third test consisted on the manufacture of simple and organic models, where the flexibility of curvature of the parts was tested.

Finally, a last test was performed with a higher degree of requirement than the previous ones. The Alveolus Block (Figure 5) is a regular base model and multiple conical openings where their union gives rise to a unique model.

These experiments were successful in verifying the ability of mixture B of being used in LDM to manufacture models in some degree of complexity.

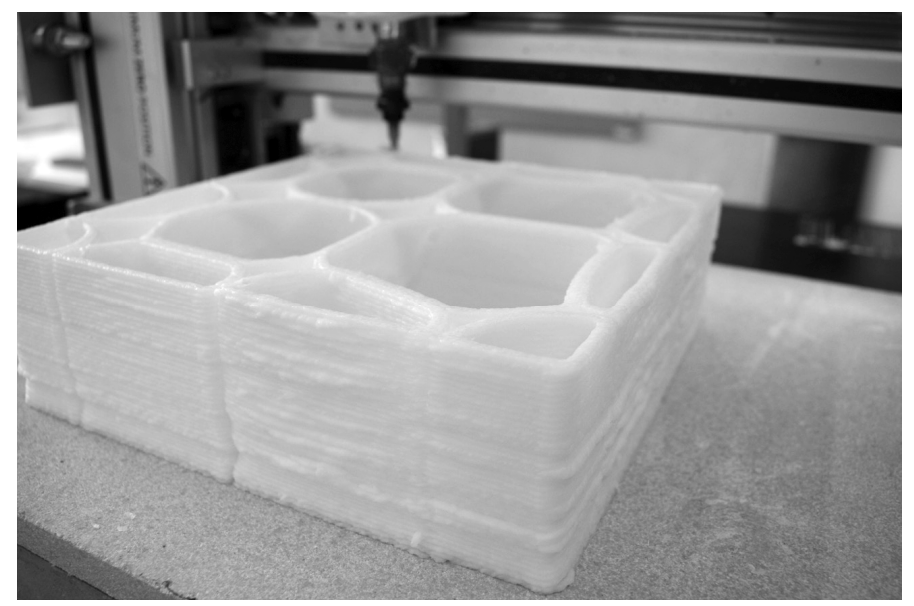

Figure 5.Alveolus block print with mixture B. 
The greatest disadvantage observed when using the mixture is in the drying phase, where the detachment and lamination of the walls of the objects produced is verified. The lamination is verified when the walls of the models lose water, through the drying, which causes the detachment of the surfaces.

Concluding, the greater the degree of complexity of the models manufactured, the more difficult is the response of the mixture, which consequently leads to the appearance of lamination, cracks and deformations in the model walls.

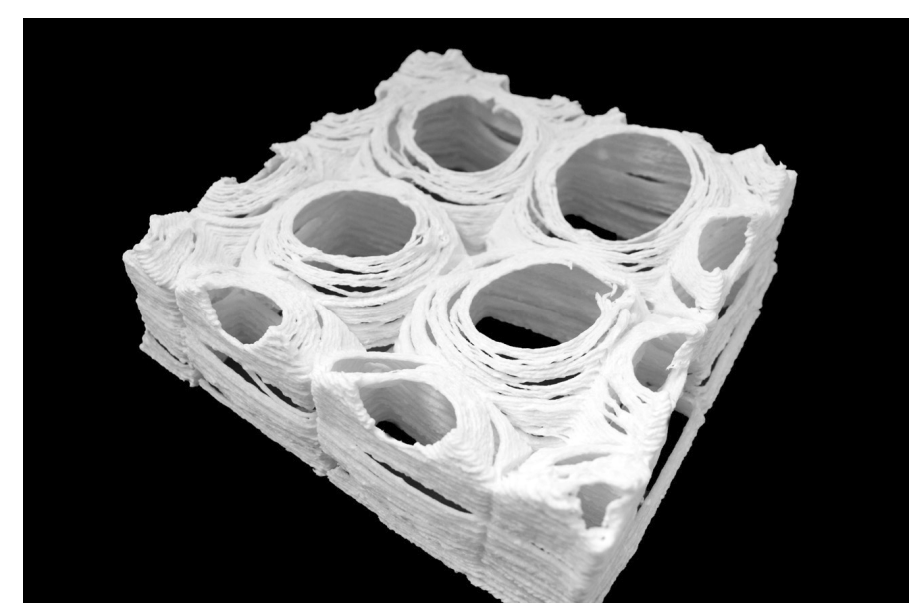

Figure 6. Lamination of the Alveolus Block walls after drying in greenhouse. Model produced with mixture B.

\subsection{Mixture B.1}

Composed by starch, cellulose pulp powder and wood fibers, the mixture B.1 is an improvement of the mixture B that came up with the purpose of solving the problems previously observed during the production and manufacture of components or models. With the same composition, with the exception of wood fibers, the main purpose when placing them in the mixture was to solve, in particular problems related to the retraction of the material. As observed in the individual study of mixture B, its workability exceeded the initially drawn targets, and the most fragile point observed was the constant lamination and detachment between the different layers that make up the manufactured model. It was thought that by placing sawdust, the retraction of the material wild be lower than previously observed, by not absorbing the water, eliminating the possibility of excessive saturations. The following steps were followed:

1. The weighing of 60 grams of cellulose pulp;

2. Attach 300 grams of previously boiled starch and aggregation of both materials.

3. Weighing of 30 grams of sawdust and subsequent aggregation of composite materials.

All tests performed on the study of mixture B were repeated. The mixture B.1 shows some improvements if compared to mixture B (Figure 6 and 7). The sawdust improves the fluidity of the mixture thus allowing a more careful, clean printing and more accurate surfaces. The higher the percentage of sawdust attached, the more accurate is the fabrication process. The retraction is also attenuated by the presence of the sawdust and it is verified a considerable decrease in the detachment and lamination produced by the evaporation of the water during the drying process. Similarly to what was observed in the mixture B, the retraction was mainly noticed in the thickness of the different layers (Figure 7).

The introduction of sawdust clearly change the appearance of the printed model. The greater the amount of sawdust is present in the mixture, the darker it gets (Figure 8). The mixture B.1 allowed to produce extruded surfaces with better finish than the ones observed in the models produced with the mixture B.

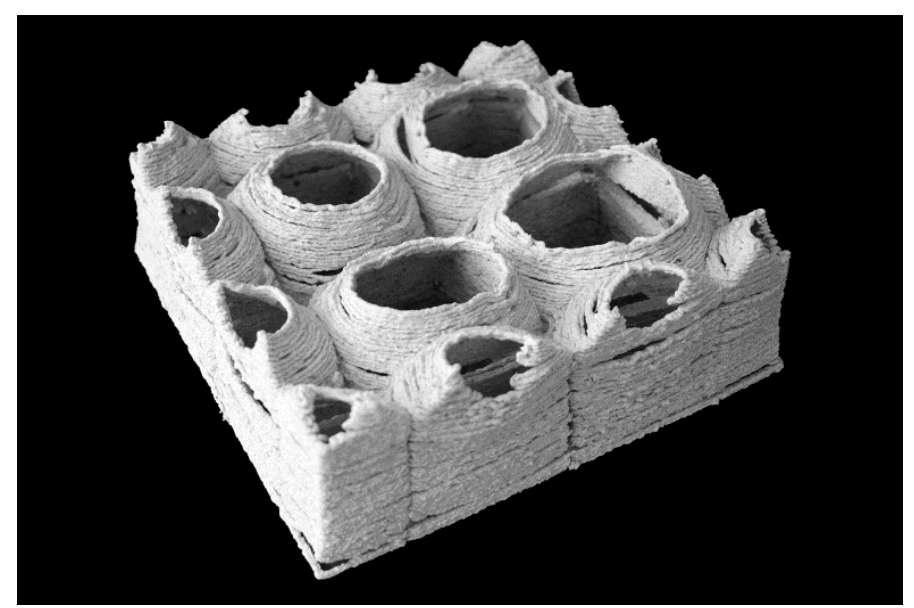

Figure 7. Reduction of the lamination observed on the walls of the Alveolus Block, when compared to the previous block, printed with the mixture B. Model produced with mixture B.1.

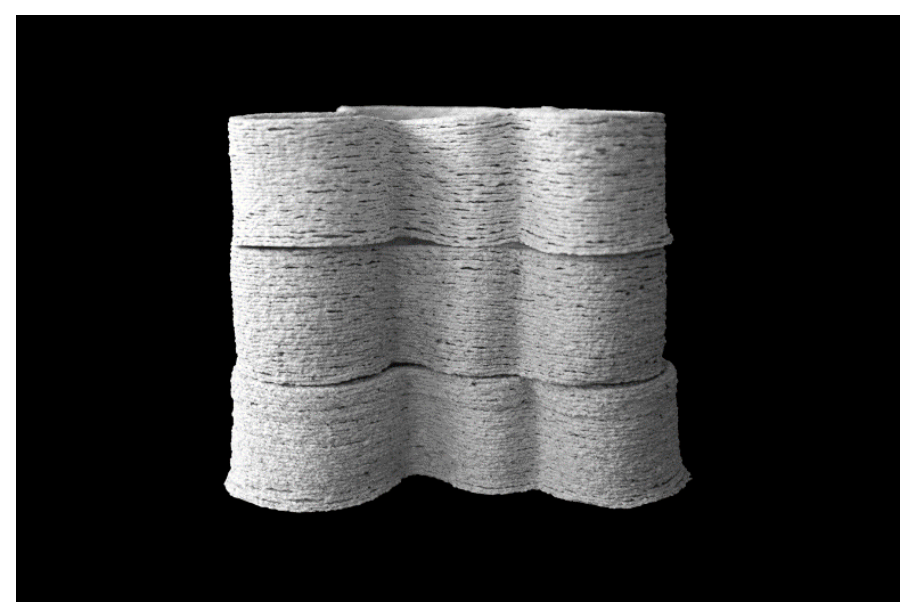

Figure 8.Final finishing of the AM Aalto pieces, printed with mixture B.1. Upper piece (15gr of sawdust), central piece $(45 \mathrm{gr}$ of sawdust) and lower piece (30gr of sawdust). 


\section{CLAY AND CELLULOSE}

\subsection{Introduction}

Clay is a mineral from sedimentary rocks that according to the place where it is found, can give rise to multiple types of clay bodies by the aggregation of other materials and minerals. Most clay bodies for architectural ceramics are earthenware and stoneware - both sedimentary clay types - as well as porcelain. These terms, used in common language to reference pottery, here designate technical expressions of the mixtures of clays and additives (Bechtold 2015).

The ceramic paste used on this study is a mixture of stoneware and water - GRES $130 \mathrm{MP}$, a ceramic paste produced and commercialized by Vicar, a Spanish company specialized in ceramic materials. With the right amount of moisture, this paste showed exceptional workability in LDM. The placement of the cellulose fibers in the paste intends to increase the potential of the mixture, evidencing a greater potential for application in architecture, through the resolution of different problems verified, when using only ceramic paste. These problems consist of:

1. Excessive retraction caused walls deformation;

2. Excessive retraction of the material taking into account the initially proposed dimensions;

3. Fissure between connections;

4. Decrease in weight of printed models.

To solve the above mentioned problems, it was believed that the placement of cellulose fibers would enhance the workability, behaviour and strength of the mixture. This research was divided into two mixtures: Mixture C, composed by clay, cellulose pulp in plates and water; Mixture D, composed by clay, cellulose pulp powder and water.

\subsection{Mixture $C$}

Composed of cellulose pulp in plates and stoneware paste. According to the tests performed in ACLab, the adequate moisture content of this stoneware paste in order to obtain models with good print quality by LDM should be between 33 and 35\% (Cruz et al. 2017). As described in mixture A, the cellulose pulp in plates, needs to suffer a previous transformation to be added to the clay. To achieve Mixture C, it's necessary to follow only two steps:

1.The weighing of 1000 grams of ceramic paste and subsequent placement of water missing;

2.The weighing of the quantity of transformed cellulose pulp and aggregation of both materials.

Given the possibility of placing several percentages of cellulose in the ceramic paste, a study was defined generating multiple mixtures until obtaining an interesting consistency for exploration:

$1.1 \mathrm{Kg}$ of ceramic paste and $25 \mathrm{gr}$ of cellulose pulp;

$2.1 \mathrm{Kg}$ of ceramic paste and $50 \mathrm{gr}$ of cellulose pulp;
$3.1 \mathrm{Kg}$ of ceramic paste and $75 \mathrm{gr}$ of cellulose pulp; $4.1 \mathrm{Kg}$ of ceramic paste and $100 \mathrm{gr}$ of cellulose pulp;

Since ceramic paste is a widely used material in the manufacture of architectural components, using the techniques of $\mathrm{AM}$, the study of the mixture $\mathrm{C}$ is based on the possibility of improvement of the clay paste. Precisely, were defined parameters of studies that consist in the workability and feasibility in the mixture when the cellulose is attached and its behaviour when applied in real manufacturing context. The first phase of work resulted in the analysis of the workability and feasibility of the mixture when attached to cellulose. In order to validate this phase, a first test consisted of the printing of a set of truncated cones, were the inclination of the walls is varied in order to observe the limitation of the curvature (Figure 9). A second test was carried out, as already stated in the study of the previously mixtures, which consisted in the manufactured of pieces inspired by geometry of the cups of the architect Alvar Aalto (Figure 10).

All tests performed with the mixture $C$ showed better results than those observed with mixture A. The presence of cellulose fibers decreases all the problems previously mentioned, except the retraction of the pieces. The retraction index verified with the ceramic paste is similar to the one observed in the mixture $\mathrm{C}$.

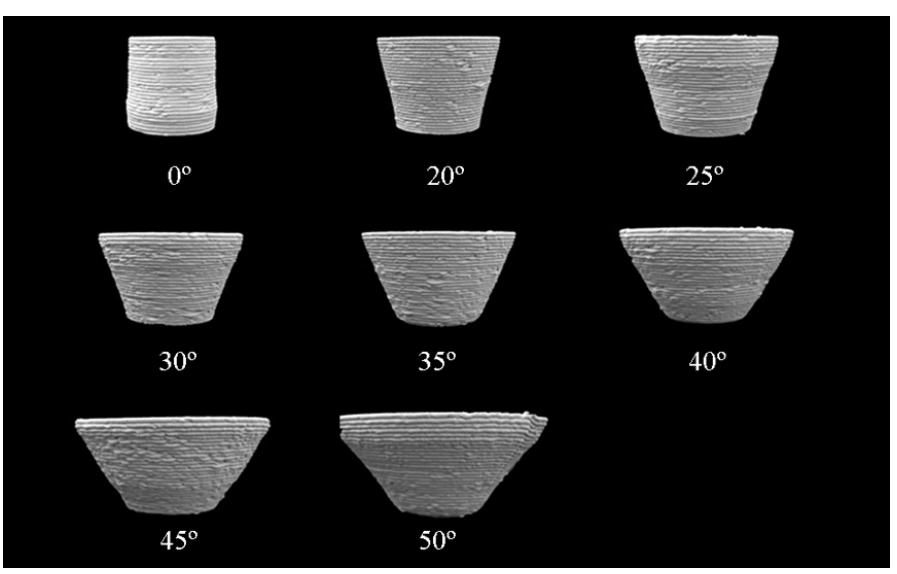

Figure 9.Final finishing of a set of truncated cones. Observation of limitation of the curvature of the mixture $\mathrm{C}$.

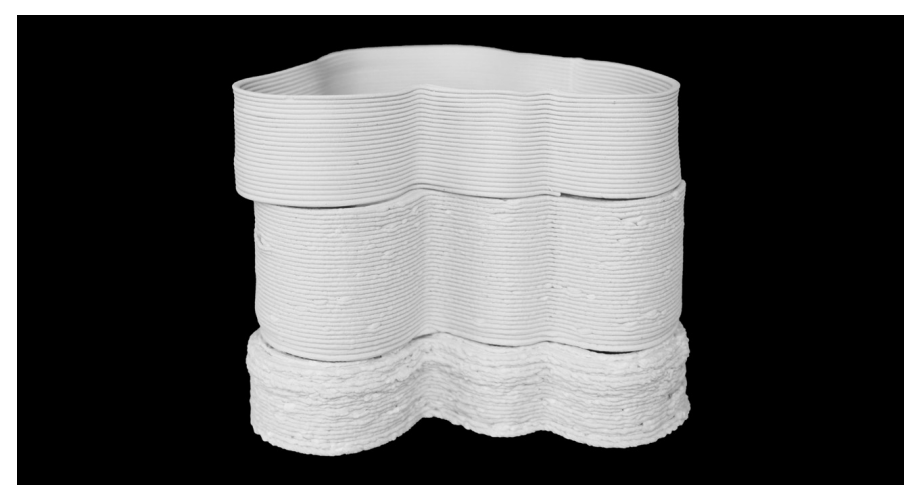

Figure 10. Top - ceramic paste, middle - ceramic paste and $25 \mathrm{gr}$ of cellulose) and bottom - ceramic paste and 75 gr of cellulose. 


\subsection{Mixture D}

As mentioned, the Mixture D was composed of cellulose pulp powder and ceramic paste. Their preparation followed the same steps of the previous mixture, except for the cellulose pulp. Given the possibility of placing several percentages of cellulose in the ceramic paste, a study was defined generating multiple mixtures until obtaining an interesting consistency for exploration:

$1.1 \mathrm{Kg}$ of ceramic paste and $10 \mathrm{gr}$ of cellulose pulp;

$2.1 \mathrm{Kg}$ of ceramic paste and $25 \mathrm{gr}$ of cellulose pulp;

$3.1 \mathrm{Kg}$ of ceramic paste and 50 gr of cellulose pulp;

$4.1 \mathrm{Kg}$ of ceramic paste and 75 gr of cellulose pulp;

When prepared the mixture D was necessary to considerate the preparation, since the powdered cellulose doesn't have moisture, when mixed with ceramic paste absorbs some of its moisture, considerably altering the expect moisture content, to ensure good workability. To control this factor, a parametric ratio was defined. For each 5 grams of powdered cellulose, was added 6 grams of water. As previously described, study parameters were defined. The first work phase, similar to described in previous subchapters, started by printing of the truncate cones.

Due to the excellent performative response of the mixture D in the first discriminated phase, the second working phase resulted in the production of the previously mentioned component - Alveolus Block.

Mixture D had a better behaviour than the observed throughout the individual study with mixture $\mathrm{C}$. With the accomplishment of these studies, the retraction index observed, with or without cellulose is almost the same, varying the greater the percentage of cellulose attached. The deformations and cracks are attenuated by the presence of cellulose in mixture.

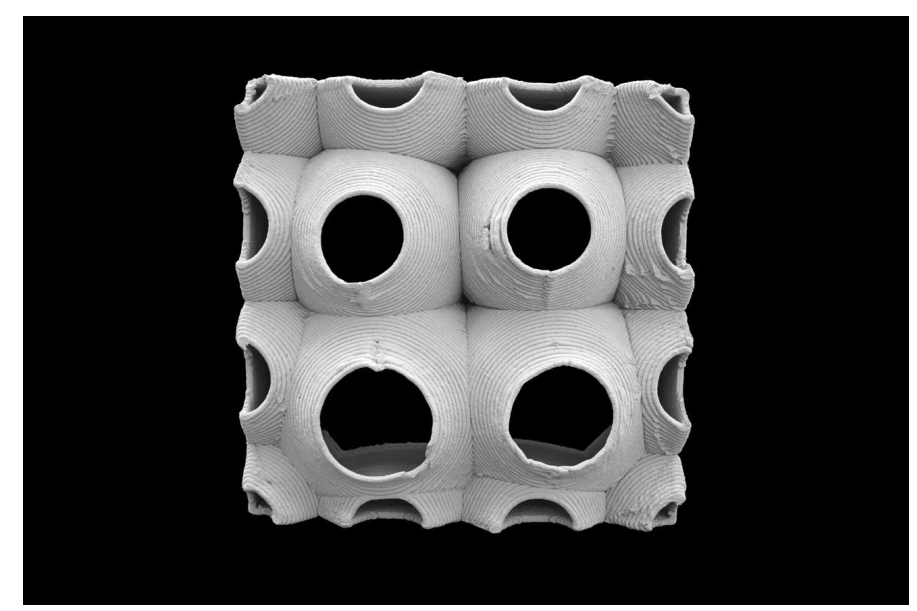

Figure 11. Alveolus Block produced with mixture D, composed by stoneware paste and 10gr of powdered cellulose.

\section{CONCLUSION}

As demonstrated throughout this paper, cellulose is a material with great potential in AM. The application of composite mixtures (cellulose and starch) in 3D printing open multiple possibilities for the development of architectural components, the Alveolus Block can be understood as an example of this if applied on the assembly of a wall or facade. By analysing the cellulose mixtures tested in the research it's possible to conclude that the mixture with the greatest potential for AM is the mixture B.1. With the introduction of sawdust, some of the negative parameters previously observed, in the study of the mixture B, were attenuated, namely the decrease of the lamination and detachment of the surfaces of the models, generating cleaner surfaces, thus allowing a greater control on the extrusion and fluidity of the mixture. Mixture A, due to the granulometry of the transformed cellulose grains, originated some problems, namely the nozzle clogging and the production of models whose surfaces look careless. By analysing the clay mixture, the presence of the cellulose pulp in the mixture was able to attenuate the problems mentioned above.

\section{ACKNOWLEDGMENTS}

This work has the financial support of the Exploratory Research Project, with the reference MITEXPL/ISF/0006/2017, MIT Portugal-2017 Program, financed by National Funds, through FCT / MCTES. We thank to RAIZ - Institute Research of Florest and Paper, for their support and partnership in this research, namely through the supply of cellulose. We are grateful to the Institute of Design of Guimarães for hosting and supporting the Advanced Ceramics R\&D Lab on the use of their facilities and equipment.

\section{REFERENCES}

Barbosa, I. \& Figueiredo, B. 2017. Optimized Brick - Print Optimization. In F.M. da Silva et al. (eds), Challenges for Technology Innovation:A agenda for the Future. 201-210. London: Taylor \& Francis Group.

Betchold, M. \& Kane, A. \& King, N. 2015. Ceramic Material Systems: in architecture and interior design. Basel: Bikhauser GmbH.

Cruz, P.J.S. Knaack, U. Figueiredo, B. Witte, D. 2017. Ceramic 3D printing - The future of brick architecture. In A. Bögle \& M. Grohmann (eds.), IASS 2017 - Interfaces: Architecture. Engineering. Science. Hamburg: HafenCity University .

Latka, J. 2017. Architecture and the Built environment - Paper in Architecture. Holland: Delft University of Technology, faculty of Architecture.

Rosenthal, M. et al. 2017. Liquid Deposition Modeling: a promising approach for 3D printing. European Journal of Wood and Wood Products. Volume 76 (Issue 2): pp 797-799. 